\title{
Protein Image Alignment via Tensor Product Cubic Spline
}

\author{
Florian A. Potra, Xing Liu
}

Apr., 2005

\begin{abstract}
The class of tensor product cubic splines is considered in an optimization process for two-dimensional polyacrylamide gel electrophoresis (2D-PAGE) image alignment problems arising in proteomics studies. Numerical results are presented along with comparisons with previous results based on piecewise affine and bilinear transformations.
\end{abstract}




\section{Introduction}

Protein image alignment problem is a very important issue in proteomics studies. Proteomics is the large-scale analysis of complex protein mixtures focusing on the qualitative and quantitative variations of protein expression levels $[18,25]$.

One of the core technologies for obtaining protein mixtures is provided by two-dimensional polyacrylamide gel electrophoresis (2D-PAGE), which was first introduced by O'Farrell [17] and Klose [13] in 1975. Along the first dimension, proteins are sorted electrophoretically according to their $\mathrm{pH}$ gradient. Each stabilizes at a point where its net charge is zero. Along the other dimension, proteins, soaked in sodium dodecyl sulphate, separate according to their molecular weight. Hence, on a 2D gel, two coordinates uniquely characterize each protein spot: its isoelectric point and its molecular weight. The separated proteins are then stained with fluorescent dyes [2] so that they are detectable for imaging. The resulting images are scanned and stored in a database. In recent years, 2D-PAGE analysis has became again one of the most active fields in proteomics studies $[3,5,24,25]$.

Experimental conditions may cause the gels to be distorted, which results in deformed images with new spot coordinates, shapes, sizes and intensity values. Some possible factors contributing to such distortions include the structure of the polyacrylamide net, the characteristics of transporting solution, the solvent conditions, and the nature of the electric field [5]. Unfortunately, these distortions cannot be avoided. Even under strictly-controlled laboratory conditions, repeated analysis of the same sample may produce non-identical gels $[21,24]$. In order to analyze 
variations in the protein gels obtained from different groups that account for biological variations attributable, for example, to a treatment we must first eliminate distortions to properly align images. The image alignment is recognized as a major bottleneck in proteomics.

Alignment of a family of gel images can be done by means of geometrical transformations applied to the coordinate domain of the images [11, 12]. This technique is also known as image warping $[7,10,21]$, or image registration $[8,9,22,23]$. The transformed images should have no (or very little) geometrical variations, so that only statistical and biological variation are observed. Most image warping methods rely on some preassigned control points or landmarks, which are a relatively small group of spots present in all the gels being compared. The landmarks should be selected so that they are evenly distributed over the gel image. The number of the landmarks should be large enough, so that they can carry enough information. However, too many landmarks will increase computational complexity and may result in over-fitting. The shape and size of the protein spots chosen as landmarks may vary significantly, but for the purpose of image alignment, only the $\mathrm{x}-\mathrm{y}$ coordinates of their centers are considered. In the remainder of the this paper, we refer to landmarks simply as the $\mathrm{x}-\mathrm{y}$ coordinates of their centers.

In our recent paper [19], we have proposed a combined forward-inverse transformation approach to align families of gel images without any pre-selected reference gel. The information contained in the landmarks of the whole family is used to create an ideal gel. Then we determine proper transformations that optimally align all the gels of the family to this ideal gel. Using the approach in [19], we have managed to align very large families of gel images. The transformations 
used in [19] are piecewise affine and bilinear transformations based on a sequence of hierarchical grids. Similar hierarchical approach has been used in $[21,23]$ for pair-wise alignment. In the present paper, we adopt the approach in [19], with the transformations replaced by the family of tensor product cubic splines. We will impose proper constraints on the transformation so that the transformations are globally $C^{2}$, in the sense that the transformation itself, the first derivatives and the second derivatives are continuous.

Tensor product splines have been used for many years $[1,4,6,16]$ for interpolation and surface fitting. However, they has never been used in in connection to optimization for image processing. In contrast with traditional interpolation, where the grid points (vertices) are to be matched, we consider the grid points as part of the unknowns in a large-scale optimization problem.

In section 2 we present the construction of piecewise polynomial transformations based on segmentations of the gel images. We then discuss the tensor product cubic spline in section 3 . The combined forward-inverse transformation approach is presented in section 4 . In section 5 we evaluate the performance of the method on two protein image data sets which were also used in [19]. Some final conclusions are presented in section 6 .

\section{Segmentation of the Gel Images}

Given a collection of $\mathrm{M}$ gel images $\mathcal{I}^{(1)}, \mathcal{I}^{(2)}, \ldots, \mathcal{I}^{(M)}$, with $\mathrm{N}$ preassigned landmarks on each image, we denote by $L_{i l}=\left(x_{i l}, y_{i l}\right)^{T}$ the $l$ th landmark on $\mathcal{I}^{(i)}, l=1, \ldots, N$. All the gel images 
in our applications are rectangles. We assume in general that $\mathcal{I}^{(i)}$ is included in the rectangle $\Omega^{(i)}=\left[\check{\tau}_{i}^{x}, \hat{\tau}_{i}^{x}\right] \times\left[\check{\tau}_{i}^{y}, \hat{\tau}_{i}^{y}\right]$. In contrast with the traditional pair-wise alignment approach, we want to construct a set of ideal landmarks $L_{l}, l=1, \ldots, N$ an ideal gel image $\mathcal{I}$, along with a set of geometric transformations $T^{(i)}: \Omega^{(i)} \rightarrow \mathbb{R}^{2}$, as the solutions obtained from the following optimization problem.

$$
\begin{array}{cc}
\min _{T^{(i)}, L_{l}} \varphi & \\
\text { subject to } & \left\|T^{(i)}\left(L_{i l}\right)-L_{l}\right\|_{\infty} \leq \varepsilon, \quad i=1, \ldots, M, l=1, \ldots, N, \\
& \left\|L_{l}-\frac{1}{M} \sum_{i=1}^{M} L_{i l}\right\|_{\infty} \leq \delta,
\end{array} \quad l=1, \ldots, N,
$$

where the objective function $\varphi$ is constructed in such a way as to maximize the smoothness of the transformations, and $\varepsilon$ and $\delta$ are some constant parameters. The first constraint in (2.1) is used to ensure that the transformed landmarks $T^{(i)}\left(L_{i l}\right)$ are close enough (within $\varepsilon$ pixels) to the ideal landmark $L_{l}$, and the second constraint is used to set the ideal landmark $L_{l}$ close (within $\delta$ pixels) to the center of the corresponding group of landmarks $L_{i l}, i=1, \ldots, M$.

As suggested in $[19,20]$, in order to avoid the drawbacks and limitations of global transformations, we consider piecewise low order polynomial transformations based on segmentations of the gel images as follows. Rectangle $\Omega^{(i)}$ is divided into $p_{i} \times q_{i}$ equal sub-rectangles

$$
\Omega_{j k}^{(i)}=\left\{v=\left(\begin{array}{l}
x \\
y
\end{array}\right) \in R^{2}, s_{j}^{(i)} \leq x \leq s_{j+1}^{(i)}, t_{k}^{(i)} \leq y \leq t_{k+1}^{(i)}\right\}, j=1, \ldots, p_{i}, k=1, \ldots, q_{i},
$$

with vertices $v_{j k}^{(i)}=\left(s_{j}^{(i)}, t_{k}^{(i)}\right)^{T}$, where

$$
s_{j}^{(i)}=\check{\tau}_{i}^{x}+(j-1) \Delta x^{(i)}, j=1, \ldots, p_{i}+1,
$$




$$
t_{k}^{(i)}=\check{\tau}_{i}^{y}+(k-1) \Delta y^{(i)}, k=1, \ldots, q_{i}+1
$$

and

$$
\Delta x^{(i)}=\frac{\hat{\tau}_{i}^{x}-\check{\tau}_{i}^{x}}{p_{i}}, \quad \Delta y^{(i)}=\frac{\hat{\tau}_{i}^{y}-\check{\tau}_{i}^{y}}{q_{i}}
$$

On each sub-rectangle $\Omega_{j k}^{(i)}$, we define a local transformation

$$
T_{j k}^{(i)}(x, y)=\left(\begin{array}{c}
f_{j k}^{(i)}(x, y) \\
g_{j k}^{(i)}(x, y)
\end{array}\right): \Omega_{j k}^{(i)} \rightarrow R^{2} .
$$

We then define the global transformation by combining all the local transformations:

$$
T^{(i)}(x, y)=\left(\begin{array}{c}
f^{(i)}(x, y) \\
g^{(i)}(x, y)
\end{array}\right)=\left(\begin{array}{c}
\sum_{j=1}^{p_{i}} \sum_{k=1}^{q_{i}} \omega_{j k}^{(i)}(x, y) f_{j k}^{(i)}(x, y) \\
\sum_{j=1}^{p_{i}} \sum_{k=1}^{q_{i}} \omega_{j k}^{(i)}(x, y) g_{j k}^{(i)}(x, y)
\end{array}\right),
$$

where

$$
\omega_{j k}^{(i)}(x, y)=\left\{\begin{array}{cc}
1 & \text { if }(x, y) \in \Omega_{j k}^{(i)} \\
0 & \text { otherwise }
\end{array} .\right.
$$

In $[19,20]$, we have managed to use piecewise affine transformations and piecewise bilinear transformations to align families of 2D-gels images. The transformations were constructed in such a way that they are globally continuous. A question arose naturally - how to obtain classes of globally $C^{1}$ or even $C^{2}$ transformations? In the following sections, tensor product cubic splines will be constructed in such a way that the global transformation is $C^{2}$ with optimized curvature. 


\section{Tensor Product Cubic Splines}

A general form of tensor product splines is given by

$$
\sum_{i=0}^{m} \sum_{j=0}^{n} V_{i j} B_{i}(x) C_{j}(y)
$$

where $V_{i j}$ are parameters and $B_{i}(x)$ and $C_{j}(y)$ are base functions. A typical representative of tensor product splines is the class of tensor product B-splines where the base function is a B-spline function $[4,6,16]$. The simplest form of tensor product splines is with linear base functions [4]. This is in fact the piecewise bilinear transformation used in $[19,20,21,23]$. By choosing cubic polynomial base functions in (3.8), we obtain tensor product cubic splines. Although tensor product cubic splines have been used before $[1,16]$ for interpolation and surface fitting, they have not been used in an optimization process for image alignment. In the present section, we aim to construct globally $C^{2}$ tensor product cubic splines by choosing proper cubic base functions.

A tensor product cubic spline is decided by a set of tensor product cubic transformations through (2.6). On each sub-rectangle, a tensor product cubic transformation can be defined as

$$
T_{j k}^{(i)}(x, y)=\left(\begin{array}{c}
f_{j k}^{(i)}(x, y) \\
g_{j k}^{(i)}(x, y)
\end{array}\right)=\left(\begin{array}{c}
\sum_{\lambda=0}^{3} \sum_{\gamma=0}^{3} a_{\lambda \gamma}^{i j k} x^{\lambda} y^{\gamma} \\
\sum_{\lambda=0}^{3} \sum_{\gamma=0}^{3} b_{\lambda \gamma}^{i j k} x^{\lambda} y^{\gamma}
\end{array}\right)
$$

We note there are 16 parameters in the transformation on each dimension. As suggested in [19], using these coefficients to define the transformation is not efficient. We thus reformulate the transformation using a unique set of base functions $h_{00}, h_{01}, h_{10}$, and $h_{11}$ that satisfies the 
following conditions.

$$
\begin{array}{llll}
h_{00}(0)=1, & h_{00}(1)=0, & h_{00}^{\prime}(0)=0, & h_{00}^{\prime}(1)=0, \\
h_{10}(0)=0, & h_{10}(1)=1, & h_{10}^{\prime}(0)=0, & h_{10}^{\prime}(1)=0, \\
h_{01}(0)=0, & h_{01}(1)=0, & h_{01}^{\prime}(0)=1, & h_{01}^{\prime}(1)=0, \\
h_{11}(0)=0, & h_{11}(1)=0, & h_{11}^{\prime}(0)=0, & h_{11}^{\prime}(1)=1 .
\end{array}
$$

That is, for $\mu, \nu, \iota, \sigma, \in\{0,1\}$, we have

$$
h_{\mu \nu}^{(\iota)}(\sigma)=\delta_{\mu \sigma} \delta_{\nu \iota}
$$

where

$$
\delta_{i j}=\left\{\begin{array}{ll}
1 & \text { if } \quad i=j \\
0 & \text { otherwise }
\end{array} .\right.
$$

We could use standard polynomial interpolation techniques such as divided difference, to obtain the base functions:

$$
\begin{aligned}
& h_{00}(x)=2 x^{3}-3 x^{2}+1, \\
& h_{10}(x)=-2 x^{3}+3 x^{2}, \\
& h_{01}(x)=x^{3}-2 x^{2}+x, \\
& h_{11}(x)=x^{3}-x^{2} .
\end{aligned}
$$

This is in fact the special case of the cubic Hermite basis [4].

We are now ready to re-formulate the tensor product cubic transformation using the above base functions. We omit the index $i$ for simplicity, and obtain

$$
f(x, y)=\sum_{j=1}^{p} \sum_{k=1}^{q} \omega_{j k}(x, y) \sum_{\iota, \sigma, \mu, \nu \in\{0,1\}} f_{j+\iota, k+\sigma}^{\mu \nu} h_{\iota \mu}\left(\frac{x-x_{j}}{x_{j+1}-x_{j}}\right) h_{\sigma \nu}\left(\frac{y-y_{k}}{y_{k+1}-y_{k}}\right),
$$




$$
g(x, y)=\sum_{j=1}^{p} \sum_{k=1}^{q} \omega_{j k}(x, y) \sum_{\iota, \sigma, \mu, \nu \in\{0,1\}} g_{j+\iota, k+\sigma}^{\mu \nu} h_{\iota \mu}\left(\frac{x-x_{j}}{x_{j+1}-x_{j}}\right) h_{\sigma \nu}\left(\frac{y-y_{k}}{y_{k+1}-y_{k}}\right),
$$

where $f_{j+\iota, k+\sigma}^{\mu \nu}, \iota, \sigma, \mu, \nu \in\{0,1\}$ are 16 free parameters defining the corresponding tensor product cubic transformation, and likewise for $g_{j+\iota, k+\sigma}^{\mu \nu}$. Moreover, we note that because the base functions satisfy condition (3.10), the following relations hold for any value of the parameters

$$
\frac{\partial^{\mu+\nu} f}{\partial x^{\mu} \partial y^{\nu}}\left(x_{j+\iota}, y_{k+\sigma}\right)=f_{j+\iota, k+\sigma}^{\mu \nu} \quad \frac{\partial^{\mu+\nu} g}{\partial x^{\mu} \partial y^{\nu}}\left(x_{j+\iota}, y_{k+\sigma}\right)=g_{j+\iota, k+\sigma}^{\mu \nu}, \quad \iota, \sigma, \mu, \nu \in\{0,1\}
$$

Let us now analyze the conditions we need to impose on the parameters to ensure global $C^{2}$ property for the tensor product cubic spline. Since each tensor product cubic transformation is $C^{2}$ on the corresponding local rectangular area, it is enough to check the $C^{2}$ property on the common edges and vertices of adjacent rectangles. For simplicity we consider a typical interior vertex $v=(\xi, \tau)$. Let $\Omega_{1}, \Omega_{2}, \Omega_{3}$, and $\Omega_{4}$ be the 4 rectangles adjacent to $v$. Let us denote by $f_{1}, f_{2}, f_{3}, f_{4}$ the horizontal component of the local transformations on defined on the rectangles $\Omega_{1}, \Omega_{2}, \Omega_{3}$, and $\Omega_{4}$, respectively. That is, $f_{i}=\left.f\right|_{\Omega_{i}}, i=1,2,3,4$. We also denote by $\Delta_{i j}$ the common edge of rectangles $\Omega_{i}$ and $\Omega_{j}$, as shown in Figure 3.1.

Let us consider as an example the horizontal common edge $\Delta_{14}$ in Figure 3.1. We denote by $v^{\prime}=(\zeta, \tau)$ the other end point on $\Delta_{14}$

We first check the $C^{0}$ property on $\Delta_{14}$. Since $y=\tau$ is fixed on $\Delta_{14}, f(x, y)$ is a polynomial of order 3 with respect to $x$. We note that a polynomial of order 3 is completely determined by two functions values and two derivative values. At the two end points of $\Delta_{14}$, the following 


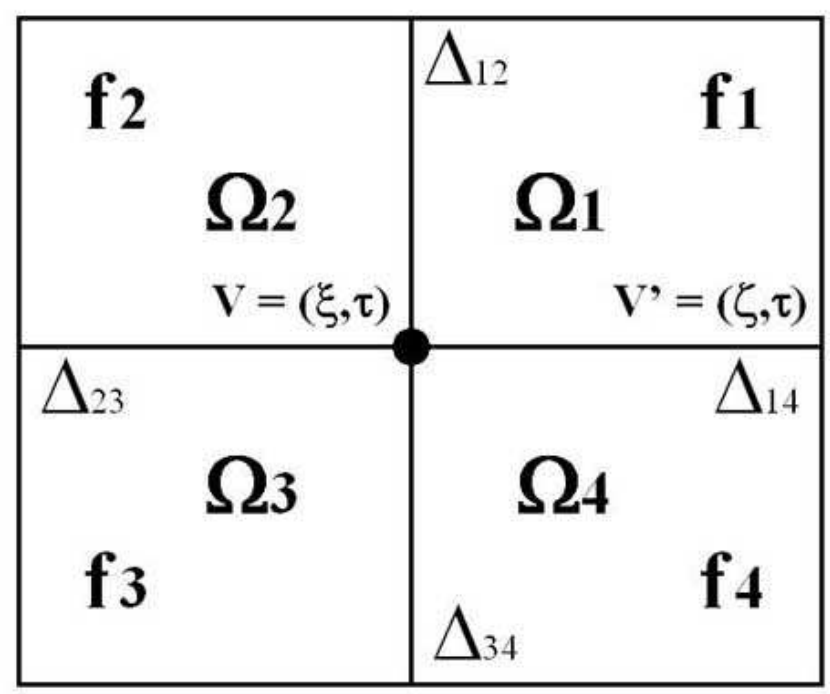

Figure 3.1: a typical interior vertex

equalities always hold

$$
f_{1}(\xi, \tau)=f_{4}(\xi, \tau), f_{1}(\zeta, \tau)=f_{4}(\zeta, \tau), \frac{\partial}{\partial x} f_{1}(\xi, \tau)=\frac{\partial}{\partial x} f_{4}(\xi, \tau), \frac{\partial}{\partial x} f_{1}(\zeta, \tau)=\frac{\partial}{\partial x} f_{4}(\zeta, \tau)
$$

This implies that

$$
f_{1}(x, \tau) \equiv f_{4}(x, \tau), \quad \forall x \in[\xi, \tau]
$$

Therefore, continuity holds on $\Delta_{14}$, for arbitrary choices of the parameters $f_{j+\iota, k+\sigma}^{\mu \nu}$.

Let us now consider the $C^{1}$ property. It follows immediately from (3.12) that

$$
\frac{\partial}{\partial x} f_{1}(x, \tau) \equiv \frac{\partial}{\partial x} f_{4}(x, \tau), \quad \forall x \in[\xi, \tau]
$$

Let us now consider $\frac{\partial f}{\partial y}$. We note that on $\Delta_{14}, \frac{\partial}{\partial y} f(x, y)$ is a polynomial of order 3 with respect 
to $x$. At the two end points of $\Delta_{14}$, we have

$$
\begin{aligned}
\frac{\partial}{\partial y} f_{1}(\xi, \tau) & =\frac{\partial}{\partial y} f_{4}(\xi, \tau), & \frac{\partial^{2}}{\partial x \partial y} f_{1}(\xi, \tau) & =\frac{\partial^{2}}{\partial x \partial y} f_{4}(\xi, \tau) \\
\frac{\partial}{\partial y} f_{1}(\zeta, \tau) & =\frac{\partial}{\partial y} f_{4}(\zeta, \tau), & \frac{\partial^{2}}{\partial x \partial y} f_{1}(\zeta, \tau) & =\frac{\partial^{2}}{\partial x \partial y} f_{4}(\zeta, \tau)
\end{aligned}
$$

This implies

$$
\frac{\partial}{\partial y} f_{1}(x, \tau) \equiv \frac{\partial}{\partial y} f_{4}(x, \tau), \quad \forall x \in[\xi, \tau]
$$

Therefore, the $C^{1}$ property holds on $\Delta_{14}$, for arbitrary choices of the parameters $f_{j+\iota, k+\sigma}^{\mu \nu}$.

At last we want to obtain $C^{2}$ for the tensor product cubic spline. Again it follows from (3.12) that

$$
\frac{\partial^{2}}{\partial x^{2}} f_{1}(x, \tau) \equiv \frac{\partial^{2}}{\partial x^{2}} f_{4}(x, \tau), \quad \forall x \in[\xi, \tau]
$$

Moreover, it follows from (3.13) that

$$
\frac{\partial^{2}}{\partial x \partial y} f_{1}(x, \tau) \equiv \frac{\partial^{2}}{\partial x \partial y} f_{4}(x, \tau), \quad \forall x \in[\xi, \tau]
$$

Therefore it remains to analyze the continuity of $\frac{\partial^{2}}{\partial y^{2}} f(x, y)$. Once $y=\tau$ is fixed, $\frac{\partial^{2}}{\partial y^{2}} f(x, y)$ is a polynomial of order 3 with respect to $x$. We thus impose the following constraints:

$$
\frac{\partial^{2}}{\partial y^{2}} f_{1}(\xi, \tau)=\frac{\partial^{2}}{\partial y^{2}} f_{4}(\xi, \tau), \quad \frac{\partial^{2}}{\partial y^{2}} f_{1}(\zeta, \tau)=\frac{\partial^{2}}{\partial y^{2}} f_{4}(\zeta, \tau)
$$

and

$$
\frac{\partial^{3}}{\partial y^{2} \partial x} f_{1}(\xi, \tau)=\frac{\partial^{3}}{\partial y^{2} \partial x} f_{4}(\xi, \tau), \quad \frac{\partial^{3}}{\partial y^{2} \partial x} f_{1}(\zeta, \tau)=\frac{\partial^{3}}{\partial y^{2} \partial x} f_{4}(\zeta, \tau)
$$

The $C^{2}$ property is therefore enforced on $\Delta_{14}$. 
A similar analysis can be done to retrieve the conditions needed to ensure the $C^{2}$ property on a vertical common edge between two adjacent horizontal rectangles.

Let us summarize the necessary constraints to enforce the $C^{2}$ property globally. According to (3.14), constraints

$$
\frac{\partial^{2}}{\partial y^{2}} f_{1}(v)=\frac{\partial^{2}}{\partial y^{2}} f_{4}(v), \quad \frac{\partial^{2}}{\partial y^{2}} f_{2}(v)=\frac{\partial^{2}}{\partial y^{2}} f_{3}(v)
$$

are to be enforced on $\Delta_{14}$ and $\Delta_{23}$, respectively. We note that the corresponding equalities hold automatically on $\Delta_{12}$ and $\Delta_{34}$, i.e.,

$$
\frac{\partial^{2}}{\partial y^{2}} f_{1}(v)=\frac{\partial^{2}}{\partial y^{2}} f_{2}(v), \quad \frac{\partial^{2}}{\partial y^{2}} f_{3}(v)=\frac{\partial^{2}}{\partial y^{2}} f_{4}(v)
$$

hold for any arbitrary choices of parameters. Therefore, instead of (3.16), it is enough to impose only

$$
\frac{\partial^{2}}{\partial y^{2}} f_{1}(v)=\frac{\partial^{2}}{\partial y^{2}} f_{4}(v)
$$

It follows from (3.15) that we have to impose also the following constraints to ensure the continuity of $\frac{\partial^{2}}{\partial y^{2}} f(v)$.

$$
\frac{\partial^{3}}{\partial y^{2} \partial x} f_{1}(v)=\frac{\partial^{3}}{\partial y^{2} \partial x} f_{4}(v), \quad \frac{\partial^{3}}{\partial y^{2} \partial x} f_{2}(v)=\frac{\partial^{3}}{\partial y^{2} \partial x} f_{3}(v)
$$

However, the corresponding equalities hold automatically on $\Delta_{12}$ and $\Delta_{34}$. This is because

$$
\frac{\partial^{2}}{\partial x \partial y} f_{1}(z) \equiv \frac{\partial^{2}}{\partial x \partial y} f_{2}(z), \quad \forall z \in \Delta_{12},
$$

and

$$
\frac{\partial^{2}}{\partial x \partial y} f_{3}(z) \equiv \frac{\partial^{2}}{\partial x \partial y} f_{4}(z), \quad \forall z \in \Delta_{34}
$$


We thus have

$$
\frac{\partial^{3}}{\partial y^{2} \partial x} f_{1}(v)=\frac{\partial^{3}}{\partial y^{2} \partial x} f_{2}(v), \quad \frac{\partial^{3}}{\partial y^{2} \partial x} f_{3}(v)=\frac{\partial^{3}}{\partial y^{2} \partial x} f_{4}(v)
$$

Therefore, instead of (3.18), it is enough to impose only

$$
\frac{\partial^{3}}{\partial y^{2} \partial x} f_{1}(v)=\frac{\partial^{3}}{\partial y^{2} \partial x} f_{4}(v)
$$

A similar analysis can be done for $\frac{\partial^{2}}{\partial x^{2}} f(v)$. We need to impose the following constraints to ensure the continuity of $\frac{\partial^{2}}{\partial x^{2}} f(v)$ on all four edges.

$$
\begin{gathered}
\frac{\partial^{2}}{\partial x^{2}} f_{1}(v)=\frac{\partial^{2}}{\partial x^{2}} f_{2}(v) \\
\frac{\partial^{3}}{\partial x^{2} \partial y} f_{1}(v)=\frac{\partial^{3}}{\partial x^{2} \partial y} f_{2}(v) .
\end{gathered}
$$

From the above argument, it follows that

$$
f, \frac{\partial f}{\partial x}, \frac{\partial f}{\partial y}, \frac{\partial^{2} f}{\partial x^{2}}, \frac{\partial^{2} f}{\partial y^{2}}, \frac{\partial^{2} f}{\partial x \partial y}
$$

are well defined and continuous on the four edges if we impose equality constraints (3.17), (3.19), (3.20), (3.21). Moreover, it also follows that all functions in (3.22) have the same value at the vertex $v$, which implies they are continuous at $v$. Therefore, we claim that we only need to impose constraints (3.17), (3.19), (3.20), (3.21), at any interior vertex $v$.

We next consider the constraints needed to be imposed on a vertex on the boundary. By using a similar analysis, it turns out that we need to impose constraints the (3.17), (3.19) for each vertex on a vertical boundary, where in this case, $f_{1}$ and $f_{4}$ in (3.17) and (3.19) stand for the transformations from the two (vertically) adjacent rectangles, and the constraints (3.20), (3.21) 
for each vertex on a horizontal boundary, where in this case, $f_{1}$ and $f_{2}$ in (3.20) and (3.21) stand for the transformations from the two (horizontally) adjacent rectangles.

The exact analysis can be performed on $g(x, y)$. In summary, the tensor product cubic spline is $C^{1}$ automatically due our selection of the base functions. In order to achieve $C^{2}$, we need to impose constraints the (3.17), (3.19), (3.20), and (3.21), if applicable (that is, depending on the location of the vertex). Altogether we need to impose

$$
2\left(4\left(p_{i}-1\right)\left(q_{i}-1\right)+2\left(2\left(p_{i}-1\right)+2\left(q_{i}-1\right)\right)\right)=8\left(p_{i} q_{i}-1\right)
$$

equality constraints in order to have a $C^{2}$ tensor product cubic spline $T^{(i)}$.

If we consider $T^{(i)}$ in (2.1) defined by tensor product cubic splines we obtain

$$
\begin{aligned}
& \min _{L_{l}, f_{j, k}^{(i) \mu \nu}, g_{j, k}^{(i) \mu \nu}} \sum_{i=1}^{M} \sum_{j=1}^{p_{i}+1} \sum_{k=1}^{q_{i}+1}\left(\frac{\partial^{2}}{\partial x^{2}} f\left(v_{j k}^{(i)}\right)^{2}+\frac{\partial^{2}}{\partial y^{2}} f\left(v_{j k}^{(i)}\right)^{2}+\frac{\partial^{2}}{\partial x \partial y} f\left(v_{j k}^{(i)}\right)^{2}\right. \\
& \left.+\frac{\partial^{2}}{\partial x^{2}} g\left(v_{j k}^{(i)}\right)^{2}+\frac{\partial^{2}}{\partial y^{2}} g\left(v_{j k}^{(i)}\right)^{2}+\frac{\partial^{2}}{\partial x \partial y} g\left(v_{j k}^{(i)}\right)^{2}+w\left(f\left(v_{j k}^{(i)}\right)-v_{j k}^{(i)}\right)^{2}\right) \\
& \text { s.t. } \quad \text { constraints } \quad(3.17),(3.19),(3.20),(3.21) \text {, } \\
& \left\|T_{\alpha(i, l), \beta(i, l)}^{(i)}\left(L_{i l}\right)-L_{l}\right\|_{\infty} \leq \varepsilon, \quad i=1, \ldots, M, l=1, \ldots, N, \\
& \left\|L_{l}-\frac{1}{M} \sum_{i=1}^{M} L_{i l}\right\|_{\infty} \leq \delta, \quad l=1, \ldots, N,
\end{aligned}
$$

where $(\alpha(i, l), \beta(i, l))$ is the index of the rectangle that $L_{i l}$ lies in:

$$
L_{i l} \in \Omega_{\alpha(i, l), \beta(i, l)}^{(i)},
$$

and $w$ is a constant parameter. The last term in the objective function measures the difference between $T^{(i)}$ and an identical transformation. The rest of the terms in the objective function 
are squared second order derivative information of the transformations at the vertices, which is a good measurement of the curvature of the transformation. We note that these second order derivatives, along with the constraints, can be written as linear function of the unknowns $f_{j+\iota, k+\sigma}^{(i)}, \mu \nu, g_{j+\iota, k+\sigma}^{(i) \mu \nu}$ and $L_{l}$. Therefore, (3.23) is a valid Quadratic Program (QP).

Let us now analyze the feasibility of the above QP. In our applications we take $p_{i}=q_{i}=p$, so that we have $8 M(p+1)^{2}$ unknowns. There are $8 M\left(p^{2}-1\right)$ linear equality constraints to ensure the $C^{2}$ property, and $2 M N+2 N$ equality constraints when $\varepsilon=\delta=0$. A solution in this case is clearly a feasible point for the QP with $\varepsilon \geq 0, \delta \geq 0$. In order to ensure feasibility, it suffices to have $16 M p+16 M \geq 2 M N+2 N$. In all our applications $16 M \geq 2 N$, so that the $\mathrm{QP}(3.23)$ is always feasible if $p \geq N / 8$. For instance, for a set of gel images that have about 20 landmarks on each, a $3 \times 3$ grid is enough to ensure feasibility.

\section{Algorithm}

We now describe the algorithm. We use the combined forward-inverse transformation approach from [19]. A forward tensor product cubic spline is constructed based on a relatively sparse segmentation of the image. As a result of the forward phase, a set of ideal landmarks $L_{l}, l=$ $1, \ldots, N$, along with the approximate size of the ideal gel $\Omega=\left[\check{\tau}^{x}, \hat{\tau}^{x}\right] \times\left[\check{\tau}^{y}, \hat{\tau}^{y}\right]$, are obtained from QP (3.23). In the second phase of our algorithm, we construct an inverse tensor product cubic spline for every pair of $\mathcal{I}$ and $\mathcal{I}^{(i)}$. That is, for each source image $\mathcal{I}^{(i)}$, we construct a tensor product cubic spline $T^{(i)}: \mathcal{I} \rightarrow \mathcal{I}^{(i)}$ as the solution of an optimization problem similar to 
(3.23). For simplicity, we omit the index $i$ and describe this process below.

The rectangle $\Omega$ is divided into $p \times q$ equal sub-rectangles

$$
\Omega_{j k}=\left\{z=\left(\begin{array}{c}
x \\
y
\end{array}\right) \in R^{2}, s_{j} \leq x \leq s_{j+1}, t_{k} \leq y \leq t_{k+1}\right\}, j=1, \ldots, p, k=1, \ldots, q,
$$

with vertices $v_{j k}=\left(s_{j}, t_{k}\right)^{T}$, where

$$
\begin{aligned}
& s_{j}=\check{\tau}^{x}+(j-1) \Delta x, j=1, \ldots, p+1, \\
& t_{k}=\check{\tau}^{y}+(k-1) \Delta y, k=1, \ldots, q+1,
\end{aligned}
$$

and

$$
\Delta x=\frac{\hat{\tau}^{x}-\check{\tau}^{x}}{p}, \quad \Delta y=\frac{\hat{\tau}^{y}-\check{\tau}^{y}}{q}
$$

We construct a tensor product cubic spline $T: \mathcal{I} \rightarrow \mathcal{I}^{(i)}$ based on this segmentation. Following the ideas from section 3 , we impose the following inequalities

$$
\left\|T_{m(l), n(l)}\left(L_{l}\right)-L_{i l}\right\|_{\infty} \leq \epsilon, l=1, \ldots, N
$$

where $(m(l), n(l))$ are the indices of the rectangle containing $L_{l}$ :

$$
L_{l} \in \Omega_{m(l), n(l)} .
$$

The objective function and the constraints keep as before and we obtain the following QP for pair-wise alignment between $\mathcal{I}$ and $\mathcal{I}^{(i)}$.

$$
\min _{f_{j, k}^{\mu \nu}, g_{j, k}^{\mu \nu}} \sum_{j=1}^{p+1} \sum_{k=1}^{q+1}\left(\frac{\partial^{2}}{\partial x^{2}} f\left(v_{j k}\right)^{2}+\frac{\partial^{2}}{\partial y^{2}} f\left(v_{j k}\right)^{2}+\frac{\partial^{2}}{\partial x \partial y} f\left(v_{j k}\right)^{2}\right.
$$




$$
\begin{array}{cr}
\left.+\frac{\partial^{2}}{\partial x^{2}} g\left(v_{j k}\right)^{2}+\frac{\partial^{2}}{\partial y^{2}} g\left(v_{j k}\right)^{2}+\frac{\partial^{2}}{\partial x \partial y} g\left(v_{j k}\right)^{2}+w\left(f\left(v_{j k}\right)-v_{j k}\right)^{2}\right) \\
\text { s.t. } \quad & \begin{array}{c}
\text { constraints } \\
\left\|T_{m(l), n(l)}\left(L_{l}\right)-L_{i l}\right\|_{\infty} \leq \varepsilon,
\end{array}
\end{array}
$$

We adopt the multi-resolution approach $[19,20]$ in the inverse transformation phase: We construct a sequence of increasingly finer partitions $\mathcal{P}_{1}^{(i)}, \mathcal{P}_{2}^{(i)}, \ldots$ on the ideal gel $\mathcal{I}$. Partition $\mathcal{P}_{\kappa}^{(i)}$ consists in dividing $\mathcal{I}$ into $p_{i, \kappa} \times q_{i, \kappa}$ rectangles where $p_{i, 1}, p_{i, 2}, \ldots, p_{i, \kappa}, \ldots$ and $q_{i, 1}, q_{i, 2}, \ldots, q_{i, \kappa}, \ldots$ are two increasing sequences of positive integers. At iteration $\kappa$, we construct an inverse tensor product cubic spline based on partition $\mathcal{P}_{\kappa}^{(i)}$. If the stopping criteria, such as the accuracy or smoothness requirements, are not met, we proceed to the next iteration. For instance, in our application we take $p_{i, \kappa+1}=2 p_{i, \kappa}, q_{i, \kappa+1}=2 q_{i, \kappa}$, so that if the stopping criteria are not met, we divide each rectangle into 4 equal sub-rectangles and repeat the construction.

Using this combined forward-inverse transformations with the multi-resolution approach in the inverse transformation phase has certain advantages in assigning the intensity values for the transformed images and decreasing the computational cost. For a complete analysis of this approach, we refer to [19]. Our algorithm can be described as follows.

\section{Algorithm}

Given source gels $\mathcal{I}^{(i)}$ with landmarks $L_{i l}$,

(forward transformations)

compute ideal landmarks $L_{l}$ and

estimate the size $\Omega$ of ideal reference gel $\mathcal{I}$ from (3.23); 


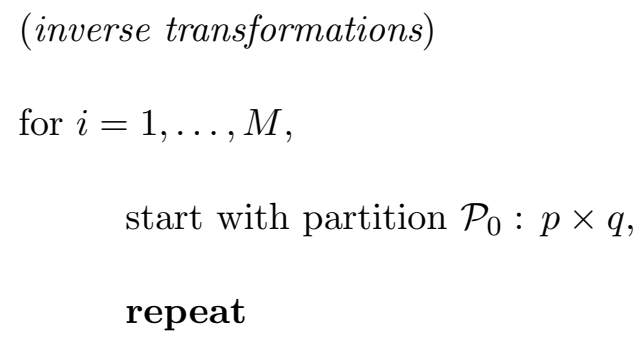

Refine the grid by setting $p=2 p, q=2 q$;

compute $\hat{\mathcal{I}}^{(i)}=T^{(i)}(\mathcal{I})$

\section{$5 \quad$ Numerical Experiments}

Our data sets were taken from a gel image database made available to public by Peter Lemkin from National Cancer Institute (http://binkley.ncifcrf.gov/users/lemkin).

Data set 1 is the Molt- 4 data

(http://www.lecb.ncifcrf.gov/2DgelDataSets/index.html\#MOLT-4). 
It contains 4 gel images $(512 \times 512$ pixels, 8-bit, 250 microns/pixel). 22 landmarks are picked on each gel.

Data set 2 is the Human leukemias data

(http://www.lecb.ncifcrf.gov/2DgelDataSets/index.html\#HEME-MALIG).

It contains 170 gel images $(512 \times 512$ pixels, 8-bit, 250 microns/pixel $)$. For more details on this data see $[14,15]$. We choose only the gel images that contain 22 landmarks on each. There are 123 such gel images in this data set. The above two data sets have been used also in [19].

We adopt the similarity measure used in [19] to measure the quality of the alignment. The normalized $l_{2}$-norm difference between two images $I$ and $J$ is given by

$$
S M=\sqrt{\frac{\sum_{i, j}\left(I_{i j}-J_{i j}\right)^{2}}{M N}},
$$

where $I_{i j}$ stands for the intensity value of the pixel $(i, j)$ on image $\mathcal{I}$, and likewise for $J_{i j}$.

The main computational tools we used is Matlab on a personal computer $\mathrm{P} 42.4 \mathrm{~Hz}$ with $768 M B$ of RAM. The QP problems are solved by the state-of-the-art software Mosek using interior-point methods. Experimental results for data set 1 are shown in table 5.1, along with the alignment results by using piecewise affine and bilinear transformations [19]. In order to have comparable results, the gray level of each image is scaled to the interval $[0,255]$ before computing the similarity measure. The forward transformations are constructed based on a $16 \times 16$ grid for the piecewise affine and bilinear transformations, and a $12 \times 12$ grid for the tensor product cubic spline. Then the inverse transformations are constructed based on a refined grid $(32 \times 32$ grid and $24 \times 24$ grid, respectively). We can see that the piecewise tensor product cubic spline 


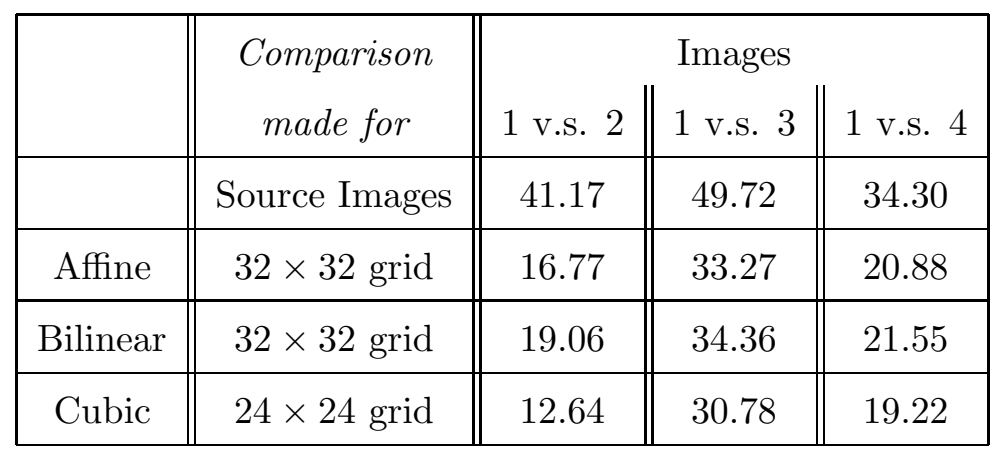

Table 5.1: A sample comparison of the similarity measures given by (5.29), for the molt-4 data.

is clearly the best choice among the three, even on a smaller sized grid. The effects of alignment are illustrated as superimposition of gel images 1 and 2 in Figure 5.2.

An important question to ask is how good is the similarity measure (5.29)? Does this measure properly reflect the quality of the alignment? Unfortunately the answer is no. Let us illustrate this in Figure 5.3. Both pictures are from the same alignment experiments, only with different pairs of images. Although the similarity between images 1 and 2 (left figure, $\mathrm{SM}=12.64$ ) is much smaller than the similarity between images 1 and 4 (right figure, $\mathrm{SM}=19.22$ ), we could not say that the alignment between images 1 and 4 is worse. In fact, we would prefer to have an alignment result like this because it is the variation that we want to observe. It seems that the difference shown in Figure 5.3 is is due to some biological variation. However, it is impossible for to tell how good the alignment result is just by looking at the similarity measure (5.29). We still need human intervention to make this kind of judgement calls. Development of a good measure of the alignment is a major challenge to address in order to fully automize the image 

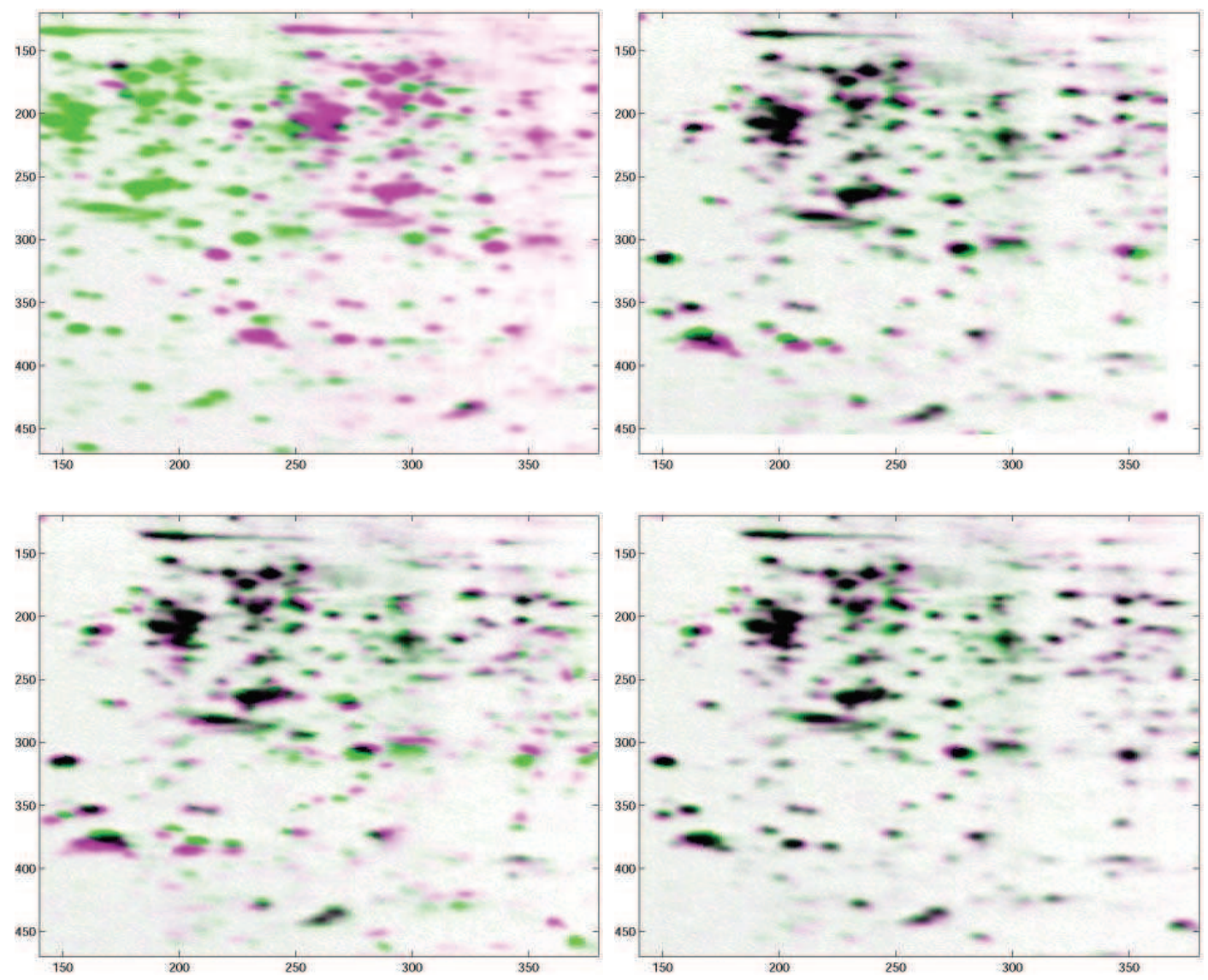

Figure 5.2: The superimposition of images 1 and 2 in the molt data. The upper left figure shows the superimposition of the original images; the upper right figure shows the superimposition of the images after affine transformations; the lower left figure shows the superimposition of the images after bilinear transformations; the lower right figure shows the superimposition of the images after cubic transformations. 

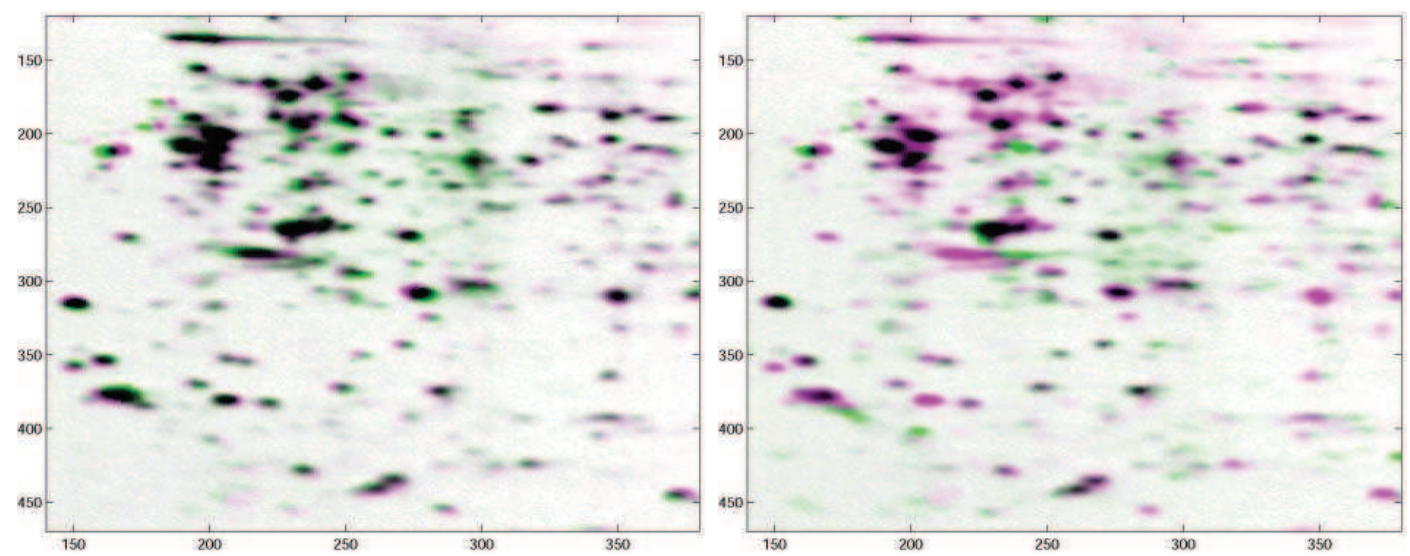

Figure 5.3: Alignment result of a pair of images in the MOLT-4 data, using tensor product cubic spline. Left figure shows the superimposition of transformed images 1 and 2. Right figure shows the the superimposition of transformed images 1 and 4 .

alignment process.

There are 123 gel images in data set 2 . We have managed to align this large collection of gel images by using a combined forward-inverse tensor product cubic spline approach. As a simple example, we show in table 5.1 a sample result of aligning image 1 (gel-HM-001.gif) and image 3 (gel-HM-003.gif), along with the alignment results by using piecewise affine and bilinear transformations. It is not very clear in this example that the tensor product cubic spline is better than the other methods. However, as suggested in [19], there may not be enough accurate landmarks to cover all over the gel images, so that an accurate alignment cannot be made in certain areas of the image. 


\begin{tabular}{|l||c||c||c||c|}
\hline & $\begin{array}{c}\text { Original } \\
\text { Images }\end{array}$ & $\begin{array}{c}\text { Inv. } 32 \times 32 \text { grid } \\
\text { Affine }\end{array}$ & $\begin{array}{c}\text { Inv. } 32 \times 32 \text { grid } \\
\text { Bilinear }\end{array}$ & $\begin{array}{c}\text { Inv. } 24 \times 24 \text { grid } \\
\text { Cubic }\end{array}$ \\
\hline Similarity Measure & 23.04 & 13.42 & 13.65 & 13.59 \\
\hline
\end{tabular}

Table 5.2: A sample comparison of the similarity measures of images 1 and 3 in the human leukemia data.

\section{Conclusion}

In the present paper, we have presented a methodology for aligning families of $2 \mathrm{D}$ gels that does not rely on choosing one of the gels as reference, but constructs an ideal gel and the corresponding warping transformations from the solution of a quadratic programming problem. We have used a combined forward-inverse tensor product cubic splines approach to align very large collections of gel images. By choosing proper base functions and constraints, the resulting tensor product cubic splines are globally $C^{2}$ with optimized curvature.

However, there are still open questions such as choosing appropriate similarity measure and performing image alignment without any pre-assigned landmarks. We will address these concerns in our future research.

This application shows the importance of optimization techniques in the field of proteomics. The development of interior-point softwares in recent years makes it possible this type of largescale proteomics studies. 


\section{References}

[1] S. A. Berger, W. C. Webster, R. A. Tapia, and D. A. Atkins. Mathematical ship lofting. Journal of Ship Research, 10(4):203-222, 1966.

[2] H. Blum, H. Beier, and H. J. Gross. Silver stain protocol. Electrophoresis, 8:93-99, 1987.

[3] K. Conradsen and J. Pedersen. Analysis of two-dimensional electrophoretic gels. Biometrics, 48:1273-1287, 1992.

[4] Carl de Boor. A practical guide to splines, volume 27 of Applied Mathematical Sciences. Springer-Verlag, New York, 1978.

[5] A. W. Dowsey, M. J. Dunn, and G.-Z. Yang. The role of bioinformatics in two-dimensional gel electrophoresis. Proteomics, 3:1567-1596, 2003.

[6] David Forsey and Richard H. Bartels. Surface fitting with hierarchical splines. ACM Transactions on Graphics, 14(2):134-161, 1995.

[7] C. A. Glasbey and K. V. Mardia. A review of image warping methods. Journal of Applied Statistics, 25:155-171, 1998.

[8] A. Goshtasby. Piecewise linear mapping functions for image registration. Pattern Recognition, 19:459-466, 1986.

[9] A. Goshtasby. Piecewise cubic mapping functions for image registration. Pattern Recognition, 20:525-533, 1987. 
[10] J. S. Gustafsson, A. Blomberg, and M. Rudemo. Warping two-dimensional electrophoresis gel images to correct for geometric distortions of the spot pattern. Electrophoresis, 23:17311744, 2002.

[11] K. Kaczmarek, B. Walczak, S. de Jong, and B.G.M. Vandeginste. Comparison of imagetransformation methods used in matching $2 \mathrm{~d}$ gel electrophoresis images. Acta Chromatographica, 13:7-21, 2003.

[12] K. Kaczmarek, B. Walczak, S. de Jong, and B.G.M. Vandeginste. Matching2d gel electrophoresis images. Journal of Chemical Information and Computer Sciences, 43:978-986, 2003.

[13] J. Klose. Protein mapping by combined isoelectric focusing and electrophoresis: a twodimensional technique. Humangenetik, 26:231-234, 1975.

[14] E. P. Lester, P. Lemkin, and L. Lipkin. A two-dimensional gel analysis of autologous t and b lymphoblastoid cell lines. Clinical Chemistry, 28(4 Pt 2):828-839, 1982.

[15] E. P. Lester, P. Lemkin, and L. Lipkin. Protein indexing in leukemias and lymphomas. Ann N Y Acad Sci., 428:158-172, 1984.

[16] Jeremiah O'Dwyer and Eberhard Vonhof. Saturable variable reluctance motor simulation using spline functions. 
[17] P.H. O'Farrell. High resolution two-dimensional electrophresis of proteins. 250:4007-4021, 1975.

[18] M.J. Page, B. Amess, C. Rohlff, C. Stubberfield, and R. Parekh. Proteomics: a major new technology for the drug discovery process. Drug Discovery Today, 4:55-62, 1999.

[19] F. A. Potra and X. Liu. Aligning families of 2d-gels by a combined multi-resolution forwardinverse transformation approach. preprint, UMBC, December 2004.

[20] F. A. Potra, X. Liu, F. Seillier-Moiseiwitsch, A. Roy, Y. Hang, M. R. Marten, B. Raman, and C. Whisnant. Protein image alignment via global and segmented local forward affine transformations. preprint, UMBC, February 2004.

[21] J. Salmi, T. Aittokallio, J. Westerholm, M. Griese, A. Rosengren, T. A. Numan, R. Lahesmaa, and O. Nevalainen. Hierarchical grid transformation for image warping in the analysis of two-dimensional electrophoresis gels. Proteomics, 2:1504-1515, 2002.

[22] Zeev Smilansky. Automatic registration for images of two-dimensional protein gels. Electrophoresis, 22:1616-1626, 2001.

[23] S. Veeser, M. J. Dunn, and G.-Z. Yang. Multiresolution image registration for twodimensional gel electrophoresis. Proteomics, 1:856-870, 2001. 
[24] T. Voss and P. Haberl. Observations on the reproducibility and matching efficiency of two-dimensional electrophoresis gels: Consequences for comprehensive data analysis. Electrophoresis, 21:3345-3350, 2000.

[25] R. Westermeier and T. Naven. Proteomics in Practice. Wiley-VCH, Weinheim, Germany, first edition, 2002. 